\title{
Clinical and molecular characterization of community-onset urinary tract infections due to extended-spectrum cephalosporin-resistant Enterobacteriaceae
}

\author{
Judith A. Anesi ${ }^{1}$, Ebbing Lautenbach ${ }^{1,2,3}$, Irving Nachamkin ${ }^{4}$, Charles Garrigan ${ }^{4}$, Warren B. \\ Bilker $^{2,3}$, Mary Wheeler ${ }^{2,3}$, Pam Tolomeo ${ }^{2,3}$, and Jennifer H. Han ${ }^{1,2,3}$ \\ ${ }^{1}$ Division of Infectious Diseases, Department of Medicine, University of Pennsylvania, \\ Philadelphia, PA, USA \\ ${ }^{2}$ Center for Clinical Epidemiology and Biostatistics, University of Pennsylvania, Philadelphia, PA, \\ USA \\ ${ }^{3}$ Department of Biostatistics and Epidemiology, University of Pennsylvania, Philadelphia, PA, USA \\ ${ }^{4}$ Department of Pathology and Laboratory Medicine, Perelman School of Medicine, University of \\ Pennsylvania, Philadelphia, PA, USA
}

\section{Abstract}

Objectives-We sought to evaluate risk factors for and molecular characteristics of communityonset extended-spectrum cephalosporin-resistant (ESC-R) Enterobacteriaceae (EB) urinary tract infections (UTIs) in a United States health system.

\begin{abstract}
Methods-A case-control study was conducted from 2010 through 2013. All patients presenting to the Emergency Department (ED) or outpatient practices with EB UTIs were included. Case patients had ESC-R EB UTIs. Control patients had ESC-susceptible EB UTIs and were matched 1:1 on study year. Risk factors for ESC-R EB UTI were assessed using multivariable conditional logistic regression. A subset of case isolates was evaluated for extended-spectrum beta-lactamases (ESBLs).
\end{abstract}

Results-302 patients with community-onset EB UTI were included, of which 151 were cases. On multivariable analysis, risk factors for ESC-R EB UTI included: trimethoprimsulfamethoxazole use in the prior 6 months (odds ratio [OR], 2.40; 95\% confidence interval [CI], $1.22-4.70 ; P=0.011$ ), older age (OR, 1.03; 95\% CI, 1.01-1.04; $P<0.001)$, diabetes (OR, 2.91; 95\% CI, 1.32-6.41; $P=0.008)$, and presentation to the ED (OR, 2.42; 95\% CI, 1.31-4.46; $P=0.005)$. The prevalence of ESBLs among 120 case isolates were: 52\% CTX-M, 29\% TEM, 20\% OXA, and $13 \%$ SHV. The prevalence of AmpC was 25\%. Pulsed-field gel electrophoresis of the CTX-M E. coli isolates showed no distinct clusters.

Conclusions-We found that use of trimethoprim-sulfamethoxazole, older age, diabetes, and presentation to the ED were associated with community-onset ESC-R EB UTI. There was a high

Corresponding author: Judith A. Anesi, MD, Division of Infectious Diseases, Department of Medicine, Hospital of the University of Pennsylvania, 3400 Spruce Street, 3 Silverstein, Suite E, Philadelphia, PA 19104, Telephone: 215-615-4724; Fax: 215-662-7611, judith.anesi@uphs.upenn.edu. 
prevalence of CTX-M among our community isolates. Further studies are needed to determine strategies to limit emergence of these organisms in the community.

\section{Keywords}

Enterobacteriaceae; Extended-spectrum cephalosporin-resistant; Extended-spectrum betalactamase; Urinary tract infection; Community onset

\section{INTRODUCTION}

Antibiotic resistance continues to emerge and threaten our antibiotic arsenal (1). Of particular concern has been the emergence of bacteria resistant to extended-spectrum cephalosporins (ESC-R), particularly among Enterobacteriaceae (EB) species. Several studies have shown that ESC-R EB infections are associated with increased morbidity, mortality, and healthcare costs $(2,3)$. The primary mechanisms causing ESC-R among EB species include production of extended-spectrum beta-lactamases (ESBLs) or an AmpC beta-lactamase (4).

Urinary tract infections (UTIs) are the most common bacterial infection among adults in the community setting (5), and recent data have demonstrated marked increases in bacterial resistance to first line antibiotics used to treat UTIs in ambulatory settings (6). Resistance has been associated with increased microbiologic and clinical failure (7).

While ESC-R EB infections in health care settings have been highlighted as an important issue for many years, the emergence of ESC-R EB infections in the community has only recently been recognized. Since its initial description, the incidence of community-onset ESC-R EB infections has increased significantly (8-12). One study performed in Switzerland reported an increase in ESC-R EB prevalence among outpatients from $0.9 \%$ in 2004 to $5.3 \%$ in 2011 (13).

Prior studies have sought to investigate risk factors for ESC-R EB infections in the community setting $(9,10,12,14-21)$. Identified risk factors have varied significantly across studies and have included: advanced age (10, 15, 16, 18, 20); diabetes mellitus (10, 22-24); prostatic disease (15); antibiotic use in the prior 3 months (10,12, 15, 17, 18, 20, 21); and prior UTIs $(15,21)$. However, these studies have had significant limitations, including small sample sizes, focus on only bloodstream infections, which are generally less common in the community setting, and focus on only one organism. Further, only one prior study evaluating risk factors for community-onset UTI with an ESC-R organism was conducted in the United States (US); this study included only ESBL-producing Escherichia coli isolates, did not differentiate between true infection and colonization, and was performed nearly 10 years ago (20), raising concerns about generalizability to the present time given marked increases in resistance over the past decade (13). Given the limitations of the one prior study done in the US, and the marked differences in antibiotic use and expected ESC-R EB epidemiology across countries, the generalizability of the prior findings to the current US population is uncertain. 
Characterizing the clinical and molecular epidemiology of ESC-R EB UTIs in the community setting is critical for identification of modifiable risk factors to curb further emergence of resistance, as well as for guiding appropriate empiric antibiotic therapy. Therefore, the objective of our study was to evaluate risk factors for community-onset ESCR EB UTIs among patients cared for in a large academic health system in the US. We also sought to evaluate the molecular epidemiology of ESC-R EB isolates causing community UTIs, including prevalence and types of ESBLs.

\section{MATERIALS AND METHODS}

\section{Study design and setting}

A case-control study was performed at two Emergency Departments (EDs) and a network of outpatient practices within the University of Pennsylvania Health System (UPHS), as follows: 1) the ED at the Hospital of the University of Pennsylvania (HUP), a 776-bed quaternary care medical center; 2) the ED at Penn Presbyterian Medical Center (PPMC), a 331-bed academic medical center, and 3) the Practice-Based Research Network (PBRN), which is a collaboration among 246 primary care physicians at community and hospitalbased practices.

\section{Study population}

The initial source population was comprised of all patients presenting to an ED or an outpatient practice with a community-onset urine culture positive for EB between December 21, 2010 and April 22, 2013. Eligible patients were identified through the HUP Clinical Microbiology Laboratory, which processes all cultures from HUP, PPMC, as well as $>90 \%$ of urine cultures from PBRN practices. A patient was designated as having a communityonset urine culture if it was obtained in the ED, outpatient practices, or within 72 hours of hospital admission from the ED or an outpatient clinic. Subsequently, only patients with a true urinary tract infection were included, as we sought to identify risk factors for ESC-R EB UTI rather than urinary colonization. A urine culture was considered indicative of an infection based on Centers for Disease Control and Prevention (CDC) definitions (25), with medical record review performed by an infectious diseases-trained physician (J.H.H.). Though these criteria were developed for surveillance purposes, they represent the only standardized definition of UTI and include the salient clinical features of UTI (25).

Case patients were defined as those with an EB UTI demonstrating resistance to an ESC (i.e., ceftriaxone or ceftazidime minimum inhibitory concentration $[\mathrm{MIC}]>1 \mu \mathrm{g} / \mathrm{mL}$ ) according to recent Clinical and Laboratory Standards Institute (CLSI) criteria (26). Control patients were those who had a UTI with ESC-susceptible EB during the study period (i.e., ceftriaxone and ceftazidime MICs $\leq 1 \mu \mathrm{g} / \mathrm{mL}$ ). Control patients were randomly selected using a computerized random number generator from the source population and matched with case patients on study year in a 1:1 ratio.

Each patient was included as a subject only once. If an ESC-R EB was isolated on multiple occasions in the same patient, only the first episode of infection was considered. The study was approved by the institutional review board of the University of Pennsylvania. 


\section{Data collection}

Data on case and control patients were abstracted from the UPHS electronic medical record system. Information was collected on demographics (e.g., age, gender, race), comorbidities (e.g., diabetes, malignancy, hemodialysis), urologic disorders (e.g., prior UTIs, urinary catheters, prostate disease within the last 6 months), recent skilled nursing facility or hospital stay, and culture location (ED versus outpatient practice).

All inpatient and outpatient antibiotic therapy in the preceding 6 months was documented. Antibiotics were classified for the purposes of analysis, as follows: penicillins (i.e. ampicillin, amoxicillin, nafcillin); extended-spectrum penicillins (amoxicillin-clavulanate, ampicillin-sulbactam, piperacillin-tazobactam); first-generation cephalosporins (i.e. cefadroxil, cefazolin, cephalexin); extended-spectrum cephalosporins (i.e. ceftriaxone, ceftazidime, cefpodoxime, cefepime); aminoglycosides; macrolides; fluoroquinolones; carbapenems; metronidazole; intravenous vancomycin; clindamycin; trimethoprimsulfamethoxazole; nitrofurantoin; and fosfomycin.

\section{Susceptibility and molecular testing of Enterobacteriaceae isolates}

Susceptibility testing of EB isolates was performed at the HUP Clinical Microbiology Laboratory. All isolates identified from study subjects were tested as part of routine care for susceptibility to other antibiotics using the semi-automated Vitek 2 identification and susceptibility system (bioMerieux, Inc., Durham, NC). Updated MIC breakpoints for ceftriaxone and ceftazidime were used without confirmatory ESBL testing according to CLSI guidelines (26).

One hundred and twenty (79.5\%) of the 151 ESC-R EB isolates were available for further microbiologic testing. These isolates underwent confirmatory ESBL testing using the double disk method, using both cefotaxime and ceftazidime (26). Real-time polymerase chain reaction (PCR) using SYBR dye for qualitative detection of TEM, SHV, OXA-1, and CTX$M$ type beta-lactamase genes, followed gel confirmation of the band sizes, were detected and grouped as previously described by the investigators was also performed (27). All strains with detectable TEM/SHV genes were further confirmed by Sanger sequencing. In addition, multiplex PCR for plasmid mediated AmpC enzymes was performed as previously described (28). Finally, the ESC-R EB isolates were screened for the presence of Klebsiella pneumoniae carbapenemase (KPC), using ertapenem susceptibility screening (29). Isolates with elevated ertapenem MICs were tested for the presence of KPC by PCR (29).

\section{Molecular typing by pulsed field gel electrophoresis (PFGE)}

The genetic relatedness of isolates was determined by molecular typing using PFGE as described previously (30). E. coli isolates that were positive for CTX-M ESBL were included in PFGE analysis. XbaI was used to prepare chromosomal digests (31). All results were analyzed using the Fingerprinting II Informatix Software version 3.0 (Bio-Rad Laboratories, Hercules, CA) and interpreted according to established criteria (32). 


\section{Statistical analysis}

Case and control patients were characterized by potential risk factors, including

demographics, comorbidities, and prior antibiotic use. Continuous variables were compared using the Student t-test or Wilcoxon rank-sum test, and categorical variables were compared using the $\chi^{2}$ or Fisher exact test. Conditional bivariable logistic regression was used to examine the relationship between each potential risk factor and ESC-R EB UTI. An odds ratio (OR) and $95 \%$ confidence interval $(\mathrm{CI})$ were calculated to evaluate the strength of any association. Multivariable conditional logistic regression was then performed, with variables from bivariable analyses with $P$ values $<0.20$ considered for inclusion in the final multivariable model. Backward stepwise selection was performed for selection of variables in the final explanatory model, with results confirmed using likelihood ratio testing (33). All analyses were performed using STATA v.13.0 (StataCorp, College Station, Texas).

\section{RESULTS}

\section{Study population}

A total of 574 patients had a community-onset urine culture with EB during the study period. Of these, 258 were excluded as they did not represent true UTI. Among the 316 community-onset UTIs, 151 were due to ESC-R EB and 165 were due to ESC-susceptible EB. One hundred fifty-one of the 165 control patients with community-onset UTI due to an ESC-susceptible EB were included and matched to the 151 case patients based on study year.

Among the entire study cohort of 302 patients, the median age was 56 years (interquartile range [IQR], 37-68), and 62 (21\%) were men. With regard to racial classification, 143 (47\%) were categorized as "white." Eighty-five (28\%) patients presented to the ED, while $217(72 \%)$ presented to an outpatient practice. The most common pathogens isolated were Escherichia coli (76\%), Klebsiella species (13\%), and Enterobacter species (9\%). Fortythree (14\%) patients required admission to a hospital (HUP or PPMC).

\section{Risk factors for community-onset UTI due to ESC-R Enterobacteriaceae}

On bivariable analysis (Table 1), several variables were noted to be significantly associated with community-onset UTI due to ESC-R EB, including older age $(P<0.001)$, male gender (OR, 2.71; 95\% CI, 1.47-5.01; $P=0.001$ ), presentation to the ED (OR, 2.39; 95\% CI, 1.38 $4.14 ; P=0.002$ ), surgery in the prior 6 months (OR, $1.79 ; 95 \% \mathrm{CI}, 1.02-3.14 ; P=0.042$ ), hospitalization in the prior 6 months (OR, 2.55; 95\% CI, 1.52-4.28; $P<0.001)$, diabetes mellitus (OR, 2.70; 95\% CI, 1.31-5.58; $P=0.007$ ), malignancy (OR, 3.50; 95\% CI, $1.73-$ 7.07; $P<0.001$ ), liver disease (OR, 3.67; 95\% CI, 1.02-13.14; $\mathrm{P}=0.046)$, and receipt within the prior 6 months of a first-generation cephalosporin (OR, 2.42; 95\% CI, 1.01-5.86; $P=0.048$ ), extended-spectrum cephalosporin ( $\mathrm{OR}, 4.75 ; 95 \% \mathrm{CI}, 1.62-13.96 ; P=0.005$ ), trimethoprim-sulfamethoxazole (SXT) $(\mathrm{OR}, 2.06 ; 95 \% \mathrm{CI}, 1.14-3.75 ; P=0.017)$, and intravenous vancomycin (OR, 10.0; 95\% CI, 1.28-78.12; $P=0.028)$.

On subsequent multivariable analysis (Table 2), receipt of SXT within the prior 6 months was significantly associated with ESC-R EB UTI (OR, 2.40; 95\% CI, 1.22-4.70; $P=0.011)$. 
Other independent risk factors associated with an ESC-R EB UTI were older age (OR, 1.03; 95\% CI, 1.01-1.04; $P<0.001$ ), diabetes mellitus (OR, 2.91; 95\% CI, 1.32-6.41; $P=0.008$ ), and presentation to the $\mathrm{ED}(\mathrm{OR}, 2.42 ; 95 \% \mathrm{CI}, 1.31-4.46 ; P=0.005)$.

\section{Susceptibility testing}

Susceptibility testing was performed on all of the EB isolates. Among the 151 ESC-R isolates, the majority were also resistance to fluoroquinolones (67\%) and SXT (57\%). In addition, $20 \%$ were resistant to aminoglycosides, and $18 \%$ were resistant to nitrofurantoin (18\%). Two percent were resistant to meropenem.

\section{Microbiologic characterization}

One hundred and twenty (79.5\%) of the 151 ESC-R EB case isolates were available for further microbiologic testing. Seventy-five $(62.5 \%)$ of these isolates were positive on double disk testing. Among the isolates that were negative on double disk testing, $31 \%$ had at least one ESBL present on PCR testing and 45\% were positive for AmpC. Among the isolates that were positive on double disk testing, $4 \%$ did not have an ESBL present on follow-up PCR testing. The prevalence of ESBLs among the ESC-R isolates were as follows (Table 3): 52\% CTX-M, 29\% TEM, 20\% OXA, and 13\% SHV. The number of ESBLs present in each ESC-R isolate varied: $28.3 \%$ had no ESBLs (specifically, no CTX-M, TEM, OXA, or SHV ESBLs), 34.2\% had one ESBL, 34.2\% had two ESBLs, 2.5\% had three ESBLs, and 0.8\% (one patient) had four ESBLs. Of the 28.3\% ESC-R isolates that did not have an ESBL, the majority $(\mathrm{n}=19 ; 56 \%)$ was positive for $\mathrm{AmpC}$.

Thirty (25\%) of the 120 characterized isolates were positive for AmpC, and five (4\%) isolates were positive for Klebsiella pneumoniae carbapenemase (KPC). Among the 120 ESC-R EB isolates, there were $57 \mathrm{E}$. coli isolates that were positive for CTX-M. There were no distinct clusters among the 57 CTX-M E. coli isolates on PFGE analysis (Supplementary Figure 1). CTX-M groups among these 57 isolates were as follows: 34 (59.6\%) were CTXM-1, 22 (38.6\%) were CTX-M-4, and 1 (1.8\%) was ungrouped.

\section{DISCUSSION}

In this case-control study, we found that recent use of SXT, older age, diabetes mellitus, and presentation to the ED (as opposed to an outpatient practice) were significant risk factors for community-onset ESC-R EB UTI in a US health system. Furthermore, we identified a high prevalence of CTX-M-producing EB causing UTIs in the community setting, with more than half of characterized ESC-R isolates positive for CTX-M. The results of our study are strengthened by a large sample size including patients who presented to two EDs and a large network of primary care practices in the US, focus on UTI which is the most common bacterial infection among adults in the community setting, and inclusion of all EB species that were resistant to extended-spectrum cephalosporins. This is only the second study to date to determine risk factors for community ESC-R EB UTI in the US and is the larger of the studies (20).

Our study showed that receipt of SXT during the prior 6 months was associated with the development of ESC-R EB UTI. Prior studies have found that antibiotic exposure is 
associated with development of ESC-R EB UTIs, including receipt of penicillins, fluoroquinolones, second- and third-generation cephalosporins, or any beta-lactam antibiotic $(10,12,15,17,18,20)$. Only one prior study has shown a specific association between SXT and ESC-R EB infection (21). The association between SXT use and ESC-R EB UTI is likely due to SXT exerting broad selection pressure on gastrointestinal flora and promoting the development of resistance. This association is particularly significant because SXT is a commonly prescribed antibiotic in the community setting; given the high prevalence of ESBL EB organisms in the community, this suggests that SXT should be used cautiously in the community, and that agents with the narrowest possible spectrum, such as nitrofurantoin, should be used when appropriate. This finding also underscores the importance of effective antibiotic stewardship measures in the community setting. Further study is indicated to assess whether decreased use of SXT can decrease the incidence of community ESC-R EB UTIs.

Similar to previous studies $(10,15,16,18)$, we found that older age was also a significant risk factor for development of an ESC-R EB UTI in the community setting. UTIs are one of the most common infections in community-dwelling older adults due to a number of agerelated risk factors, including increased rates of neurogenic bladder, increased use of urinary catheters, benign prostatic hypertrophy in men, and vaginal atrophy and increased incontinence in women (34). Furthermore, older adults may be at higher risk for colonization and subsequent infection with antibiotic-resistant organisms, including ESC-R $\mathrm{EB}$, as a result of chronic comorbidities and aging-related immunosenescence.

Comorbid diabetes mellitus was also associated with the development of community-onset ESC-R EB UTI in our study. The presence of diabetes mellitus is a well-described risk factor for infections due to ESC-R organisms (e.g., UTI, bacteremia) (10, 22-24). This elevated risk is likely related to the hyperglycemia-related impairment of immune response in diabetes mellitus as well as increased prescription of antibiotics for conditions such as asymptomatic bacteriuria (35).

The results of this study also showed that presentation to the ED (as opposed to an outpatient practice) was significantly associated with development of an ESC-R EB UTI in the community setting. Medically complex patients with greater healthcare and antibiotic exposures are more likely to present to the hospital ED, rather than an outpatient clinic, for presumed infection. It is also possible that patients with ESC-R EB UTIs were more symptomatic than those with an ESC-susceptible EB UTI and were thus more likely to present to the ED. Of note, in contrast to prior studies, our study did not show any significant association between recent hospitalization or stay in a long-term care facility and acquisition of an ESC-R EB UTI in the community $(14,19)$.

Finally, we found that there was a high prevalence (52\%) of CTX-M ESBLs among the community ESC-R EB isolates that we evaluated, as well as a high prevalence of AmpC $(25 \%)$. This is consistent with prior reports that have found an increasing prevalence of CTX-M in the community $(9,36-38)$. This high prevalence of CTX-M is concerning, given the association between CTX-M and multi-drug resistance, particularly to oral agents that are typically prescribed for community-onset UTIs. High rates of resistance to these oral 
agents were confirmed in our study, with a resistance rate of $72 \%$ to both fluoroquinolones and SXT in CTX-M-positive isolates.

There are potential limitations of our study. Misclassification is a concern in case-control studies. However, the outcome of community ESC-R EB UTI was validated through medical record review by an infectious diseases-trained physician, rather than relying on diagnostic or billing codes. While we evaluated $80 \%$ of the 151 ESC-R EB isolates, lack of characterization of the entire group may have impacted estimates of ESBL prevalence and antibiotic susceptibility profiles. Finally, the present study was conducted in a single healthcare system, and the results may not be generalizable to other dissimilar institutions.

In conclusion, the results of our study demonstrated that recent use of SXT, older age, diabetes mellitus, and presentation to the ED were significant risk factors for the development of a community-onset UTI with an ESC-R EB organism in a US health system. Further studies are needed to evaluate the clinical impact of these ESC-R EB infections, as well as to determine optimal infection control strategies to limit the spread of these highly resistant and increasingly common organisms in the community.

\section{Acknowledgments}

Funding information. This work was supported by the Agency for Healthcare Research and Quality (grant number R18-HS020002 to E.L.); the National Institutes of Health (grant numbers K24-AI080942 to E.L., K01-AI103028 to J.H.H.); and by the CDC Cooperative Agreement (grant number FOA\#CK000163 to E.L.) Epicenters for the Prevention of Healthcare Associated Infections.

\section{References}

1. Tenover FC. Development and spread of bacterial resistance to antimicrobial agents: an overview. Clin Infect Dis. 2001; 33(Suppl 3):S108-115. [PubMed: 11524705]

2. Lautenbach E, Patel JB, Bilker WB, Edelstein PH, Fishman NO. Extended-spectrum betalactamase-producing Escherichia coli and Klebsiella pneumoniae: risk factors for infection and impact of resistance on outcomes. Clin Infect Dis. 2001; 32:1162-1171. [PubMed: 11283805]

3. Schwaber MJ, Navon-Venezia S, Kaye KS, Ben-Ami R, Schwartz D, Carmeli Y. Clinical and economic impact of bacteremia with extended- spectrum-beta-lactamase-producing Enterobacteriaceae. Antimicrob Agents Chemother. 2006; 50:1257-1262. [PubMed: 16569837]

4. Bradford PA. Extended-spectrum beta-lactamases in the 21st century: characterization, epidemiology, and detection of this important resistance threat. Clin Microbiol Rev. 2001; 14:933951. table of contents. [PubMed: 11585791]

5. Simonsen L, Conn LA, Pinner RW, Teutsch S. Trends in infectious disease hospitalizations in the United States, 1980-1994. Arch Intern Med. 1998; 158:1923-1928. [PubMed: 9759689]

6. Talan DA, Krishnadasan A, Abrahamian FM, Stamm WE, Moran GJ. Group EMINS. Prevalence and risk factor analysis of trimethoprim-sulfamethoxazole- and fluoroquinolone-resistant Escherichia coli infection among emergency department patients with pyelonephritis. Clin Infect Dis. 2008; 47:1150-1158. [PubMed: 18808361]

7. Talan DA, Stamm WE, Hooton TM, Moran GJ, Burke T, Iravani A, Reuning-Scherer J, Church DA. Comparison of ciprofloxacin (7 days) and trimethoprim-sulfamethoxazole (14 days) for acute uncomplicated pyelonephritis pyelonephritis in women: a randomized trial. JAMA. 2000; 283:1583-1590. [PubMed: 10735395]

8. Pitout JD, Nordmann P, Laupland KB, Poirel L. Emergence of Enterobacteriaceae producing extended-spectrum beta-lactamases (ESBLs) in the community. J Antimicrob Chemother. 2005; 56:52-59. [PubMed: 15917288] 
9. Calbo E, Romani V, Xercavins M, Gomez L, Vidal CG, Quintana S, Vila J, Garau J. Risk factors for community-onset urinary tract infections due to Escherichia coli harbouring extended-spectrum beta-lactamases. J Antimicrob Chemother. 2006; 57:780-783. [PubMed: 16492721]

10. Colodner R, Rock W, Chazan B, Keller N, Guy N, Sakran W, Raz R. Risk factors for the development of extended-spectrum beta-lactamase-producing bacteria in nonhospitalized patients. Eur J Clin Microbiol Infect Dis. 2004; 23:163-167. [PubMed: 14986159]

11. Apisarnthanarak A, Kiratisin P, Mundy LM. Predictors of mortality from community-onset bloodstream infections due to extended-spectrum beta-lactamase-producing Escherichia coli and Klebsiella pneumoniae. Infect Control Hosp Epidemiol. 2008; 29:671-674. [PubMed: 18624669]

12. Apisarnthanarak A, Kiratisin P, Saifon P, Kitphati R, Dejsirilert S, Mundy LM. Clinical and molecular epidemiology of community-onset, extended-spectrum beta-lactamase-producing Escherichia coli infections in Thailand: a case-case-control study. Am J Infect Control. 2007; 35:606-612. [PubMed: 17980240]

13. Kronenberg A, Hilty M, Endimiani A, Muhlemann K. Temporal trends of extended-spectrum cephalosporin-resistant Escherichia coli and Klebsiella pneumoniae isolates in in- and outpatients in Switzerland, 2004 to 2011. Euro Surveill. 2013:18.

14. Ben-Ami R, Rodriguez-Bano J, Arslan H, Pitout JD, Quentin C, Calbo ES, Azap OK, Arpin C, Pascual A, Livermore DM, Garau J, Carmeli Y. A multinational survey of risk factors for infection with extended-spectrum beta-lactamase-producing enterobacteriaceae in nonhospitalized patients. Clin Infect Dis. 2009; 49:682-690. [PubMed: 19622043]

15. Azap OK, Arslan H, Serefhanoglu K, Colakoglu S, Erdogan H, Timurkaynak F, Senger SS. Risk factors for extended-spectrum beta-lactamase positivity in uropathogenic Escherichia coli isolated from community-acquired urinary tract infections. Clin Microbiol Infect. 2010; 16:147-151. [PubMed: 19689464]

16. Rodriguez-Bano J, Alcala J, Cisneros JM, Grill F, Oliver A, Horcajada JP, Tortola T, Mirelis B, Navarro G, Cuenca M, Esteve M, Pena C, Llanos AC, Canton R, Pascual A. Escherichia coli producing SHV-type extended-spectrum beta-lactamase is a significant cause of communityacquired infection. J Antimicrob Chemother. 2009; 63:781-784. [PubMed: 19223299]

17. Yilmaz E, Akalin H, Ozbey S, Kordan Y, Sinirtas M, Gurcuoglu E, Ozakin C, Heper Y, Mistik R, Helvaci S. Risk factors in community-acquired/onset urinary tract infections due to extendedspectrum beta-lactamase-producing Escherichia coli and Klebsiella pneumoniae. J Chemother. 2008; 20:581-585. [PubMed: 19028620]

18. Rodriguez-Bano J, Alcala JC, Cisneros JM, Grill F, Oliver A, Horcajada JP, Tortola T, Mirelis B, Navarro G, Cuenca M, Esteve M, Pena C, Llanos AC, Canton R, Pascual A. Community infections caused by extended-spectrum beta-lactamase-producing Escherichia coli. Arch Intern Med. 2008; 168:1897-1902. [PubMed: 18809817]

19. Moor CT, Roberts SA, Simmons G, Briggs S, Morris AJ, Smith J, Heffernan H. Extendedspectrum beta-lactamase (ESBL)-producing enterobacteria: factors associated with infection in the community setting, Auckland, New Zealand. J Hosp Infect. 2008; 68:355-362. [PubMed: 18353497]

20. Banerjee R, Strahilevitz J, Johnson JR, Nagwekar PP, Schora DM, Shevrin I, Du H, Peterson LR, Robicsek A. Predictors and molecular epidemiology of community-onset extended-spectrum betalactamase-producing Escherichia coli infection in a Midwestern community. Infect Control Hosp Epidemiol. 2013; 34:947-953. [PubMed: 23917909]

21. Rogers BA, Ingram PR, Runnegar N, Pitman MC, Freeman JT, Athan E, Havers SM, Sidjabat HE, Jones M, Gunning E, De Almeida M, Styles K, Paterson DL. Australasian Society for Infectious Diseases Clinical Research N. Community-onset Escherichia coli infection resistant to expandedspectrum cephalosporins in low-prevalence countries. Antimicrob Agents Chemother. 2014; 58:2126-2134. [PubMed: 24468775]

22. Laupland KB, Church DL, Vidakovich J, Mucenski M, Pitout JD. Community-onset extendedspectrum beta-lactamase (ESBL) producing Escherichia coli: importance of international travel. J Infect. 2008; 57:441-448. [PubMed: 18990451]

23. Rodriguez-Bano J, Navarro MD, Romero L, Martinez-Martinez L, Muniain MA, Perea EJ, PerezCano R, Pascual A. Epidemiology and clinical features of infections caused by extended-spectrum 
beta-lactamase-producing Escherichia coli in nonhospitalized patients. J Clin Microbiol. 2004; 42:1089-1094. [PubMed: 15004058]

24. Briongos-Figuero LS, Gomez-Traveso T, Bachiller-Luque P, Dominguez-Gil Gonzalez M, GomezNieto A, Palacios-Martin T, Gonzalez-Sagrado M, Duenas-Laita A, Perez-Castrillon JL. Epidemiology, risk factors and comorbidity for urinary tract infections caused by extendedspectrum beta-lactamase (ESBL)-producing enterobacteria. Int J Clin Pract. 2012; 66:891-896. [PubMed: 22897466]

25. Horan TC, Andrus M, Dudeck MA. CDC/NHSN surveillance definition of health care-associated infection and criteria for specific types of infections in the acute care setting. Am J Infect Control. 2008; 36:309-332. [PubMed: 18538699]

26. CLSI. Performance Standards for Antimicrobial Susceptibility Testing M100-S20. CLSI; Wayne, PA: 2010.

27. McGettigan SE, Hu B, Andreacchio K, Nachamkin I, Edelstein PH. Prevalence of CTX-M betalactamases in Philadelphia, Pennsylvania. J Clin Microbiol. 2009; 47:2970-2974. [PubMed: 19587301]

28. Pérez-Pérez FJ, Hanson ND. Detection of plasmid-mediated AmpC beta-lactamase genes in clinical isolates by using multiplex PCR. J Clin Microbiol. 2002; 40:2153-2162. [PubMed: 12037080]

29. McGettigan SE, Andreacchio K, Edelstein PH. Specificity of ertapenem susceptibility screening for detection of Klebsiella pneumoniae carbapenemases. J Clin Microbiol. 2009; 47:785-786. [PubMed: 19144799]

30. Lautenbach E, Fishman NO, Metlay JP, Mao X, Bilker WB, Tolomeo P, Nachamkin I. Phenotypic and genotypic characterization of fecal Escherichia coli isolates with decreased susceptibility to fluoroquinolones: results from a large hospital-based surveillance initiative. J Infect Dis. 2006; 194:79-85. [PubMed: 16741885]

31. Sabbuba NA, Mahenthiralingam E, Stickler DJ. Molecular epidemiology of Proteus mirabilis infections of the catheterized urinary tract. J Clin Microbiol. 2003; 41:4961-4965. [PubMed: 14605124]

32. Tenover FC, Arbeit RD, Goering RV, Mickelsen PA, Murray BE, Persing DH, Swaminathan B. Interpreting chromosomal DNA restriction patterns produced by pulsed-field gel electrophoresis: criteria for bacterial strain typing. J Clin Microbiol. 1995; 33:2233-2239. [PubMed: 7494007]

33. Mickey RM, Greenland S. The impact of confounder selection criteria on effect estimation. Am J Epidemiol. 1989; 129:125-137. [PubMed: 2910056]

34. Hu KK, Boyko EJ, Scholes D, Normand E, Chen CL, Grafton J, Fihn SD. Risk factors for urinary tract infections in postmenopausal women. Arch Intern Med. 2004; 164:989-993. [PubMed: 15136308]

35. Muller LM, Gorter KJ, Hak E, Goudzwaard WL, Schellevis FG, Hoepelman AI, Rutten GE. Increased risk of common infections in patients with type 1 and type 2 diabetes mellitus. Clin Infect Dis. 2005; 41:281-288. [PubMed: 16007521]

36. Woodford N, Ward ME, Kaufmann ME, Turton J, Fagan EJ, James D, Johnson AP, Pike R, Warner M, Cheasty T, Pearson A, Harry S, Leach JB, Loughrey A, Lowes JA, Warren RE, Livermore DM. Community and hospital spread of Escherichia coli producing CTX-M extended-spectrum betalactamases in the UK. J Antimicrob Chemother. 2004; 54:735-743. [PubMed: 15347638]

37. Munday CJ, Whitehead GM, Todd NJ, Campbell M, Hawkey PM. Predominance and genetic diversity of community- and hospital-acquired CTX-M extended-spectrum beta-lactamases in York, UK. J Antimicrob Chemother. 2004; 54:628-633. [PubMed: 15294889]

38. Ho PL, Poon WW, Loke SL, Leung MS, Chow KH, Wong RC, Yip KS, Lai EL, Tsang KW. group Cs. Community emergence of CTX-M type extended-spectrum beta-lactamases among urinary Escherichia coli from women. J Antimicrob Chemother. 2007; 60:140-144. [PubMed: 17496058] 


\section{Table 1}

Bivariable conditional logistic regression of risk factors for community-onset UTI due to extended-spectrum cephalosporin-resistant Enterobacteriaceae.

\begin{tabular}{|c|c|c|c|c|}
\hline Variable & Cases $(\mathrm{n}=151)^{a}$ & Controls $(\mathrm{n}=151)^{a}$ & OR $(95 \% \mathrm{CI})$ & $P$ value \\
\hline Age, median years (IQR) & $60(46-70)$ & $49(27-64)$ & ---- & $<0.001$ \\
\hline Male sex & $43(28)$ & $19(13)$ & $2.71(1.47-5.01)$ & 0.001 \\
\hline Emergency Department & $55(36)$ & $30(20)$ & $2.39(1.38-4.14)$ & 0.002 \\
\hline Surgery in prior 6 months & $36(24)$ & $21(14)$ & $1.79(1.02-3.14)$ & 0.042 \\
\hline Hospitalization in prior 6 months & $66(44)$ & $35(23)$ & $2.55(1.52-4.28)$ & $<0.001$ \\
\hline Non-white race & $78(52)$ & $81(54)$ & $0.92(0.59-1.45)$ & 0.729 \\
\hline UTI in prior 6 months & $65(43)$ & $57(38)$ & $1.22(0.79-1.90)$ & 0.372 \\
\hline Urinary catheter & $14(9)$ & $8(5)$ & $1.86(0.74-4.65)$ & 0.187 \\
\hline Rehabilitation or SNF stay in prior 6 months & $9(6)$ & $3(2)$ & $4.00(0.85-18.84)$ & 0.080 \\
\hline Prostate disease (if male) & $20(47)$ & $6(32)$ & $1.00(0.06-15.99)$ & $>0.999$ \\
\hline \multicolumn{5}{|l|}{ Comorbidities } \\
\hline Diabetes mellitus & $31(21)$ & $14(9)$ & $2.70(1.31-5.58)$ & 0.007 \\
\hline Malignancy & $39(26)$ & $14(9)$ & $3.50(1.73-7.07)$ & $<0.001$ \\
\hline Hemodialysis & $5(3)$ & $1(1)$ & $5.00(0.58-42.80)$ & 0.142 \\
\hline Solid organ transplant & $13(9)$ & $6(4)$ & $2.17(0.82-5.70)$ & 0.117 \\
\hline Respiratory disease $b$ & $29(19)$ & $17(11)$ & $1.80(0.96-3.38)$ & 0.068 \\
\hline Liver disease ${ }^{c}$ & $11(7)$ & $3(2)$ & $3.67(1.02-13.14)$ & 0.046 \\
\hline \multicolumn{5}{|l|}{ Medications $^{d}$} \\
\hline Penicillins & $8(5)$ & $4(3)$ & $2.33(0.60-9.02)$ & 0.220 \\
\hline Extended-spectrum penicillins & $12(8)$ & $5(3)$ & $2.75(0.88-8.64)$ & 0.083 \\
\hline First-generation cephalosporins & $18(12)$ & $8(5)$ & $2.42(1.01-5.86)$ & 0.048 \\
\hline Extended-spectrum cephalosporins & $19(13)$ & $4(3)$ & $4.75(1.62-13.96)$ & 0.005 \\
\hline Fluoroquinolones & $97(64)$ & $94(62)$ & $1.08(0.69-1.69)$ & 0.733 \\
\hline SXT & $36(24)$ & $19(13)$ & $2.06(1.14-3.75)$ & 0.017 \\
\hline Nitrofurantoin & $28(19)$ & $26(17)$ & $1.09(0.62-1.91)$ & 0.773 \\
\hline Fosfomycin & $2(1)$ & $1(1)$ & $2.00(0.18-22.06)$ & 0.571 \\
\hline Vancomycin & $10(7)$ & $1(1)$ & $10.0(1.28-78.12)$ & 0.028 \\
\hline Aminoglycosides & $3(2)$ & $1(1)$ & $3.00(0.31-28.84)$ & 0.341 \\
\hline Macrolides & $2(1)$ & $4(3)$ & $0.50(0.09-2.73)$ & 0.423 \\
\hline Clindamycin & $4(3)$ & $5(3)$ & $0.80(0.21-2.98)$ & 0.739 \\
\hline Doxycycline & $5(3)$ & $3(2)$ & $1.67(0.40-6.97)$ & 0.484 \\
\hline Carbapenems & $5(3)$ & $1(1)$ & $5.00(0.58-42.80)$ & 0.142 \\
\hline Metronidazole & $6(4)$ & $3(2)$ & $2.00(0.50-7.97)$ & 0.327 \\
\hline
\end{tabular}

Infect Control Hosp Epidemiol. Author manuscript; available in PMC 2017 December 01. 
$c_{\text {Hepatitis or cirrhosis. }}$

$d_{\text {Receipt in the prior } 6 \text { months. }}$

Abbreviations: CI, confidence interval; IQR, interquartile range; OR, odds ratio; SNF, skilled nursing facility; SXT, trimethoprimsulfamethoxazole; UTI, urinary tract infection. 


\section{Table 2}

Multivariable conditional logistic regression model of risk factors for community-onset UTI due to extendedspectrum cephalosporin-resistant Enterobacteriaceae.

\begin{tabular}{lcc}
\hline Variable & OR $(\mathbf{9 5 \%}$ CI $)$ & $\boldsymbol{P}$ value \\
\hline Age & $1.03(1.01-1.04)$ & $<0.001$ \\
\hline Presentation to ED & $2.42(1.31-4.46)$ & 0.005 \\
\hline Diabetes mellitus & $2.91(1.32-6.41)$ & 0.008 \\
\hline SXT receipt within prior 6 months & $2.40(1.22-4.70)$ & 0.011 \\
\hline
\end{tabular}

Abbreviations: CI, confidence interval; ED, Emergency Department; OR, odds ratio; SXT, trimethoprim-sulfamethoxazole; UTI, urinary tract infection. 


\section{Table 3}

Prevalence of ESBLs, AmpC, and KPC among cases.

\begin{tabular}{ll}
\hline ESBLs & $\begin{array}{l}\text { Prevalence }^{a} \\
\mathbf{N}(\%)\end{array}$ \\
\hline CTX-M (CTX-M-1, CTX-M-4) & $62(52)$ \\
\hline TEM (TEM-1, TEM-12) & $35(29)$ \\
\hline OXA (OXA-1) & $24(20)$ \\
\hline SHV (SHV 1, 11, 12, 28) & $15(13)$ \\
\hline ampC (CIT, FOX, DHA) & $30(25)$ \\
\hline KPC (KPC-1) & $5(4)$ \\
\hline
\end{tabular}

${ }^{a}$ For 120 case isolates. The presence of ESBLs are not mutually exclusive (e.g., SHV and TEM can co-occur), so the total prevalence will be greater than $100 \%$.

Abbreviations: ESBL, extended-spectrum beta-lactamase; KPC, Klebsiella pneumoniae carbapenemase. 